\title{
A Photofission Delayed $\gamma$-ray Spectra Calculation Tool for the Conception of a Nuclear Material Characterization Facility
}

\author{
D. BERNARD ${ }^{*}$, O. SEROT ${ }^{*}$, E. SIMON* ${ }^{*}$ L. BOUCHER ${ }^{*}$ and S. PLUMERI ${ }^{+}$
}

\begin{abstract}
The photon interrogation analysis is a nondestructive technique allowing to identify and quantify fissile materials in nuclear waste packages. This paper details an automatic procedure which has been developed to simulate the delayed $\gamma$-ray spectra for several actinide photofissions. This calculation tool will be helpful for the fine conception (collimation, shielding, noise background optimizations, etc.) and for the on-line analysis of such a facility.
\end{abstract}

Index Terms-Photonic Interrogation, Nuclear Material Characterization, Photofission Yields, Delayed $\gamma$, Decay Data.

\section{INTRODUCTION}

$\mathrm{T}$ HIS paper proposes a new tool to help the conception of a new facility in order to detect and distinguish actinides in a waste package. The technique used is based on the analysis of delayed photons emitted after photon-burst-induced fissions. Because of the difference on fission yields, the delayed photons spectra are a signature of different actinides, if any.

The first section will present the overall studied facility for the photon beam inducing fissions and the delayed gamma detection set-up. The second part will be devoted to the specific developed tool, called DEGAS (Delayed Gamma Spectrum), to obtain $\gamma$ spectra (including fission yields and decay data libraries, Bateman depletion equations solving involving numerical issues and the verification of the whole procedure). At the end, we will propose an automated tool to select specific $\gamma$-ray lines ratios to detect a specific actinide in the waste package.

\section{THE EXAMINATION FACILITY}

This facility, under design phase, has to detect the amount of actinides and to distinguish uranium from plutonium species in nuclear waste packages. Some of these waste

Paper submitted to ANIMMA conference for proceedings and to IEEE transactions in June 2017. This work was supported by the CEA/DEN and ANDRA/R\&D collaborative project DISN/ANDRA/CHK/A-CARAC-01-12.

${ }^{*}$ CEA/DEN/DER\&DTN Cadarache, F-13108 Saint-Paul-Lez-Durance, France, corresponding authors: david.bernard@cea.fr, olivier.serot@cea.fr, eric.simon@cea.fr, lionel.boucher@cea.fr.

ANDRA, 1-7 rue Jean Monnet, F-92290 Châtenay-Malabry, France, corresponding author : stephane.plumeri@andra.fr. packages are made of concrete in a big cylindrical container of more than $800 \mathrm{~L}$. Despite of the photon attenuation through concrete, the photon interrogation is one of the most ambitious ways to interrogate the waste package. The photon pulsed beam is generated by an electron convertor made of tungsten or other metal (copper, silicon,...) allowing the Bremsstrahlung emission. The LINAC maximum energy is supposed to be flexible between 15 to $25 \mathrm{MeV}$. During this irradiation phase (roughly 2 to 3 hours), the waste package is axially rotating to make uniform the irradiation dose. The beam is then stopped and after a reasonable decay time ( 1 to 5 minutes), High Purity Germanium detectors are deployed all around the package to permit the photon spectrum acquisition for a large duration (around 2 to 3 hours). This photon spectrum contains delayed $\gamma$-rays and X-rays emitted by the fission products decay. Its analysis then gives the quantitative information on the fissile mass present in the waste container.

The next sections will be devoted to the developed tools to perform the fine conception of this facility.

\section{BATEMAN EQUATIONS}

The irradiation phase and the cooling/acquisition phase will be described sequentially.

\section{A. Irradiation Phase \\ 1) Statement of the problem}

$\gamma$-induced fission of actinides leads to around 1000 independent fission products for which we have to calculate the atomic number densities $\boldsymbol{N}_{\boldsymbol{i}}(\boldsymbol{t})$, at a given time $\boldsymbol{t}$, by solving the coupled Bateman equations [1] :

$$
\begin{gathered}
\frac{d N_{i}}{d t}=-\lambda_{i} N_{i}+\sum_{j \neq i} B_{j \rightarrow i} \lambda_{j} N_{j} \\
+\int Y_{f \rightarrow i}\left(E_{\gamma}\right) N_{f} \sigma_{(\gamma, f)}\left(E_{\gamma}\right) \phi\left(E_{\gamma}, r, \omega t\right) d E_{\gamma}
\end{gathered}
$$

The first two terms are associated to decay probabilities $(\boldsymbol{\lambda})$ and branching ratios $(\boldsymbol{B})$ for the disappearance and creation of the $\boldsymbol{i}$ fission product (based on the specific JEFF-3.1.1/Decay Data Library [2]). The last term involves the production rate by folding (with respect to the incident photon energy $\boldsymbol{E}_{\boldsymbol{\gamma}}$ ) the photofission cross section $(\boldsymbol{\sigma})$ of the fissile actinide $f$ times its 
independent fission yield $(\boldsymbol{Y})$ to the incident photon flux $\boldsymbol{\phi}$. The $\boldsymbol{N}_{\boldsymbol{i}}$ number density is calculated at the distance $\boldsymbol{r}$ (location of the fission event) from the center of the cylindrical waste package. The latter is rotating during this irradiation phase with a period $T=2 \pi / \omega$ close to 2 to 3 minutes.

In order to solve equation (1), we assume first the decoupling in energy, space and time of the photon incident flux $\phi\left(E_{\gamma}, \boldsymbol{r}, \boldsymbol{\omega} t\right)=\boldsymbol{\varphi}\left(\boldsymbol{E}_{\gamma}\right) \times \boldsymbol{\psi}(\boldsymbol{r}) \times \boldsymbol{T}(\boldsymbol{t})$. In addition, since the rotation period of the waste package is much lower than the irradiation time, the time dependent production term is reduced to the averaged value. By this way, equation (1) is transformed into the following equation:

$$
\frac{d N_{i}}{d t} \cong-\lambda_{i} N_{i}+\sum_{j \neq i} B_{j \rightarrow i} \lambda_{j} N_{j}+\widetilde{Y}_{f \rightarrow i} \times \overline{\tau_{f}}(r) \times m_{f}
$$

Where:

- $\widetilde{\boldsymbol{Y}}_{f \rightarrow i}$ stands for the energy-averaged independent isotopic fission product yield $\boldsymbol{i}$ for an actinide $\boldsymbol{f}$,

- $\quad \overline{\boldsymbol{\tau}_{\boldsymbol{f}}}(\boldsymbol{r})$ its specific fission rate (relative to the mass $\boldsymbol{m}_{\boldsymbol{f}}$ that we look for).

For a given fissile isotope and by identification of equation (1) and equation (2), the effective yield $\widetilde{\boldsymbol{Y}}_{f \rightarrow \boldsymbol{i}}$ is defined as:

$$
\widetilde{Y}_{f \rightarrow i}=\frac{\int_{0}^{E_{\gamma}^{e n d}} Y_{f \rightarrow i}\left(E_{\gamma}\right)\left[\sigma_{(\gamma, f)}\left(E_{\gamma}\right) \varphi\left(E_{\gamma}\right)\right] d E_{\gamma}}{\int_{0}^{E_{\gamma}^{e n d}}\left[\sigma_{(\gamma, f)}\left(E_{\gamma}\right) \varphi\left(E_{\gamma}\right)\right] d E_{\gamma}}
$$

Where:

- The incident $\gamma$-spectrum $\boldsymbol{\varphi}\left(\boldsymbol{E}_{\gamma}\right)$ is calculated with MCNPX [3] and depends on the electron/photon conversion target and on the maximum energy of the LINAC (see figure 1).

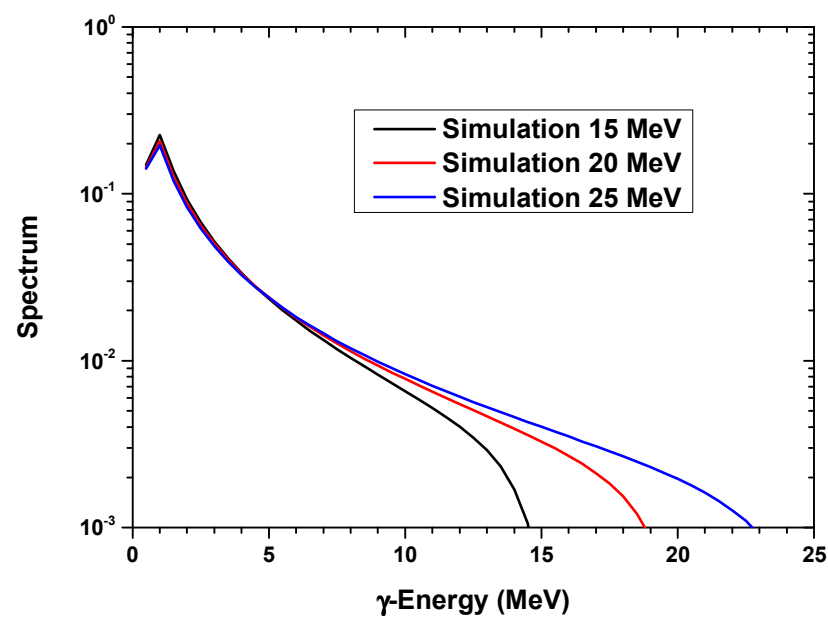

Fig. 1. Incident photon spectra in the direction of the electron beam. Calculations done with MCNPX (target conversion is W).

The maximum spectrum energy $\boldsymbol{E}_{\gamma}^{\text {end }}$ will be fixed to $15 \mathrm{MeV}$ for the whole paper but can be modified by the user as an input data of the developed tool DEGAS.
- The photofission cross sections $\boldsymbol{\sigma}_{(\gamma, f)}\left(\boldsymbol{E}_{\gamma}\right)$ are taken from the ENDF/B-VII.1 library [4] for major actinides $\left({ }^{235,238} \mathrm{U},{ }^{238,239,240,241} \mathrm{Pu},{ }^{241} \mathrm{Am}\right)$ and from the TENDL2015 library [5] for ${ }^{242} \mathrm{Pu}$ and ${ }^{242 \mathrm{~m}} \mathrm{Am}$ :

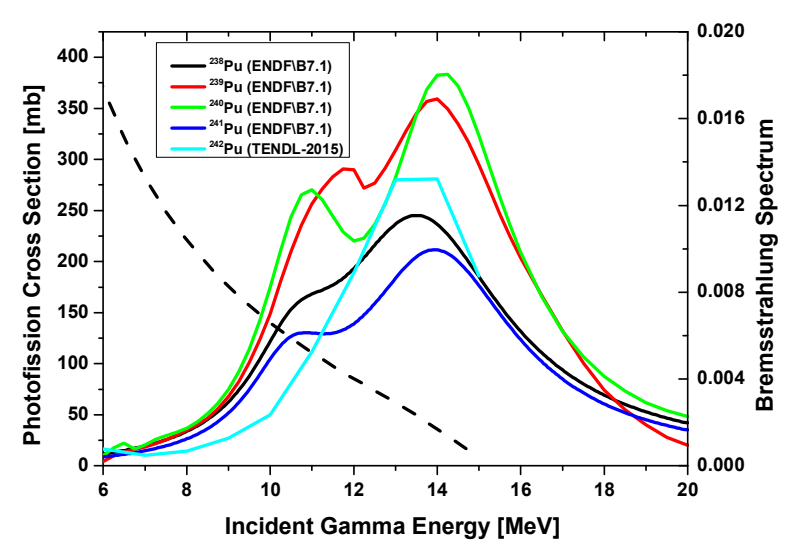

Fig. 2. Evaluated photofission cross sections (left axis) with incident $\gamma$ spectrum (dashed line, right axis).

- The independent fission product yield for a given fissioning nucleus at a given $\boldsymbol{E}_{\boldsymbol{\gamma}}$ incident energy, $\boldsymbol{Y}_{\boldsymbol{f} \rightarrow \boldsymbol{i}}\left(\boldsymbol{E}_{\boldsymbol{\gamma}}\right)$, is determined from a Monte Carlo code called GEF (acronym for GEneral description of Fission observables) [6]. This code first calculates fission fragment yields before prompt neutron emission, from the concept of fission modes developed by Brosa [7]. These fission modes correspond to the various valleys observed in the deformation potential energy surface of the fissioning nucleus. When the nucleus starts its deformation from the ground state up to the scission point, it follows one of those valleys with a given probability. The depth and width of each mode are determined in order to deduce the main properties of the fission fragments (yield, deformation at scission, kinetic energy...). In a second step, the total excitation energy available at scission is calculated and shared between the two nascent fragments. Finally, thanks to a statistical treatment, the emission of prompt neutrons and prompt gammas are simulated to predict their properties (energy spectrum, multiplicity ...). In this way, the GEF code is able to calculate almost all fission observables, in particular the isotopic and isomeric yields after prompt neutron evaporation which we are needed for. It should also be noted that GEF can simulate any type of fission: spontaneous fission, neutron-induced fission, photofission, etc. Nevertheless, in the case of photofission, the current version of the code does not allow taking into account the second and third chance of fission which should not significantly affects the present results. Examples of effective independent mass yields obtained with a $15 \mathrm{MeV}$ LINAC and a $\mathrm{W}$ conversion target are shown in figure 3. One of the main fission characteristics is clearly visible: the stabilization of the mass yield in the 
heavy mass region (around the mass 140) and consequently a shift of the mass yield in the light mass region from one fissioning nucleus to another, which ensures the conservation of the fissioning nucleus mass (removing the prompt neutron multiplicity).
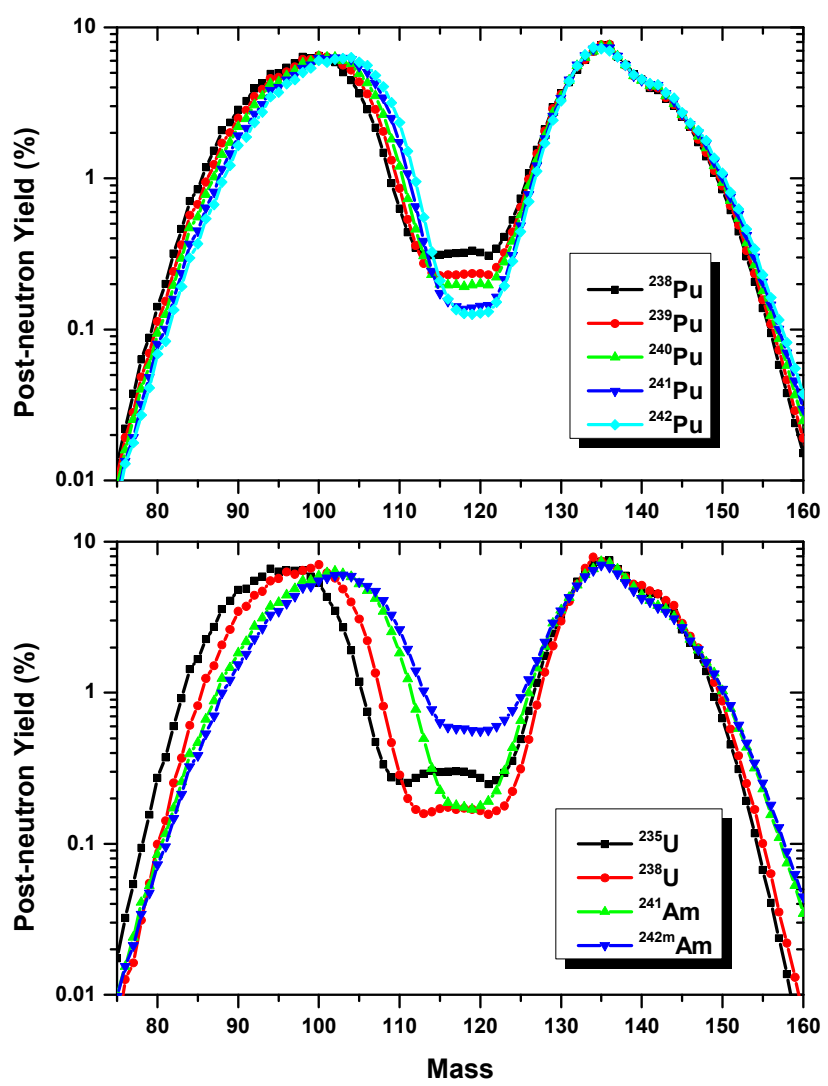

Fig. 3. Independent effective fission $(15 \mathrm{MeV}+\mathrm{W})$ mass yields obtained from the GEF code (version 2015 V2.2) [6]

The average excitation energy available for a given fissioning nucleus can be easily calculated:

$$
<E *>=\frac{\int_{0}^{E_{\gamma}^{e n d}} E_{\gamma} \sigma_{(\gamma, f)}\left(E_{\gamma}\right) \varphi\left(E_{\gamma}\right) d E_{\gamma}}{\int_{0}^{E_{\gamma}^{e n d}}\left[\sigma_{(\gamma, f)}\left(E_{\gamma}\right) \varphi\left(E_{\gamma}\right)\right] d E_{\gamma}}
$$

These energies are reported in Tab. 1 for all fissioning nuclei investigated in the present work.

\section{TABLE I}

SURVEY OF THE AVERAGE EXCITATION ENERGY AVAILABLE (IN MEV) FOR THE FISSIONING NUCLEI, CONSIDERING AN INCIDENT $\gamma$ - SPECTRUM GENERATED WITH A W CONVERSION TARGET AND A 15MEV ENERGY OF THE LINAC.

\begin{tabular}{|c|c|c|c|c|c|c|c|c|}
\hline${ }^{238} \mathrm{Pu}$ & ${ }^{239} \mathrm{Pu}$ & ${ }^{240} \mathrm{Pu}$ & ${ }^{241} \mathrm{Pu}$ & ${ }^{242} \mathrm{Pu}$ & ${ }^{235} \mathrm{U}$ & ${ }^{238} \mathrm{U}$ & ${ }^{241} \mathrm{Am}$ & ${ }^{242 \mathrm{~m}} \mathrm{Am}$ \\
\hline $\mathbf{1 0 . 6}$ & $\mathbf{1 1 . 1}$ & $\mathbf{1 0 . 9}$ & $\mathbf{1 0 . 8}$ & $\mathbf{1 1 . 1}$ & $\mathbf{1 1 . 3}$ & $\mathbf{1 1 . 0}$ & $\mathbf{1 0 . 9}$ & $\mathbf{1 1 . 9}$ \\
\hline
\end{tabular}

The second factor $\overline{\boldsymbol{\tau}_{f}}(\boldsymbol{r})$ of equation (2) is a time averaged fission rate while the waste package is rotating in front of the electron/photon beam. With a simple hypothesis of the flux attenuation as a function of the square of the distance, one can write:

$$
\overline{\tau_{f}}(\boldsymbol{r})=\tau_{f}(0) \times\left[1-\left(\frac{r}{d_{\gamma}+R_{P}}\right)^{2}\right]
$$

Where the user's input data $r, d_{\gamma}, R_{P}$ stand for the distance of the fissile material from the center of the waste package, the distance between the conversion target and the package surface and finally, the package radius, respectively.

\section{2) Solving the Bateman equations}

For a given fissile nucleus amount, we will solve the previously described equation 2 . The user has to fix a given mass $\boldsymbol{m}_{\boldsymbol{f}}$ and the location $r$ in the waste package for this particular isotope. The boundary conditions impose that no fission product are present before the irradiation in the waste package, $\boldsymbol{N}_{\boldsymbol{i}}(\boldsymbol{t}=\mathbf{0})=\mathbf{0}$.

We decide to use the DARWIN code package [8] to solve such equations, because of its robustness but also because it contains already preprocessed nuclear data libraries. This code package is able to solve non homogeneous differential equation but the source term has to be associated to neutron induced production and not to photon induced reactions. One can analytically demonstrate that solving the following homogeneous and time-iterative system (see lower case for "homogeneous" number densities $\mathbf{n}_{\mathbf{i}}$ ):

$$
\left\{\begin{array}{c}
\frac{d n_{i}}{d t}=-\lambda_{i} n_{i}+\sum_{j \neq i} B_{j \rightarrow i} \lambda_{j} n_{j} \\
n_{i}(x \cdot \delta t)=\widetilde{Y}_{f \rightarrow i} \cdot \overline{\tau_{f}}(r) \cdot m_{f} \cdot \delta t
\end{array}\right.
$$

leads to the following actual number densities which solve equation (2):

$$
N_{i}\left(\Delta t_{\text {irrad }}\right)=\sum_{x} n_{i}\left(\Delta t_{i r r a d}-x . \delta t\right)
$$

Our developed DEGAS tool is using a MATLAB ${ }^{\circledR}$ [9] script which reads first the output file from the GEF simulation $\left(\widetilde{\boldsymbol{Y}}_{\boldsymbol{f} \rightarrow \boldsymbol{i}}\right)$, produces and submits a DARWIN input file (owing to the user specifications $-\boldsymbol{m}_{f}, \Delta \boldsymbol{t}_{\text {irrad }}{ }^{-}$) in a second step, and then calculates the atomic number densities at the end of the irradiation phase: $\boldsymbol{N}_{\boldsymbol{i}}\left(\Delta \boldsymbol{t}_{\text {irrad }}\right)$ from equation (6).

\section{B. Acquisition Phase}

Once the irradiation beam is stopped, one has to calculate the integrated activity for each fission product during the cooling/acquisition phase. To do so, a similar equation as equation (6) has to be solved, where the number densities obtained at the end of the previous phase are taken as initial conditions:

$$
\left\{\begin{array}{c}
\frac{d n_{i}}{d t}=-\lambda_{i} n_{i}+\sum_{j \neq i} B_{j \rightarrow i} \lambda_{j} n_{j} \\
n_{i}(t=0)=N_{i}\left(\Delta t_{i r r a d}\right)
\end{array}\right.
$$

The DEGAS script generates a second input file for the DARWIN code package and calculates the delayed photon integrated source, starting after the HPGe deployment $\left(\Delta \boldsymbol{t}_{\boldsymbol{G} e}\right.$ 
around 1 to 5 minutes) up to the acquisition time $\left(\Delta t_{a c q}\right)$ as follows:

$$
S_{\gamma}\left(E_{\gamma}\right)=\sum_{i} \int_{t=\Delta t_{G e}}^{\Delta t_{a c q}+\Delta t_{G e}} I_{\gamma, i}\left\{\lambda_{i} \times n_{i}(t)\right\} d t
$$

where the continuous sum is approximated by a discrete sum with the same number of time steps as the previous irradiation phase. $\boldsymbol{I}_{\boldsymbol{\gamma}, \boldsymbol{i}}$ stands for the $\gamma$ or X-rays intensities of the transitions associated to the decay of the $i$ fission product.

\section{VERIFICATION OF THE WHOLE CALCULATION PROCEDURE}

A first simple verification case consists in producing one atom of ${ }^{135} \mathrm{Xe}$ per second during $\Delta \boldsymbol{t}_{\text {irrad }}=1 \mathrm{~h}$ and calculating the emitted $\gamma$-spectrum during $\Delta \boldsymbol{t}_{\boldsymbol{a c q}}=1 \mathrm{~h}$ after the HPGe deployment $\Delta \boldsymbol{t}_{\boldsymbol{G e}}=1 \mathrm{~s}$. The analytical solution of equation (2) gives 3466.88 nuclei at the end of irradiation phase. The DEGAS script gives the following results according to numerous times steps number (Tab. II).

TABLE II

VALIDATION OF THE IRRADIATION PHASE CALCULATION.

\begin{tabular}{|c|c|}
\hline Number of integration time steps & $\begin{array}{c}\text { End of irradiation } \\
\text { atomic number densities of }{ }^{135} \mathrm{Xe}\end{array}$ \\
\hline 10 & 3453.75 \\
\hline 50 & 3464.26 \\
\hline 100 & $\mathbf{3 4 6 5 . 5 7}$ \\
\hline 1000 & 3466.75 \\
\hline 10000 & 3466.87 \\
\hline
\end{tabular}

We estimate that 100 time steps for solving equations (6) and (7) are a good compromise versus the calculation time consuming.

The analytical value by solving equations (8) and (9) leads to 227.87 emitted $\gamma$ for the $250 \mathrm{keV}$ emission. This exact value is obtained with the iterative calculation scheme. See figure 4 for the full $\gamma$-spectrum :

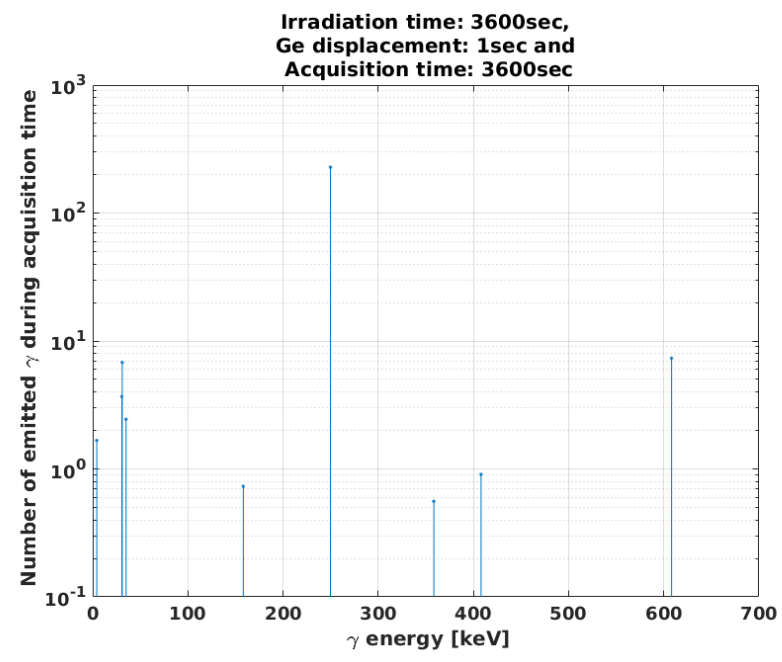

Fig. 4. Delayed $\gamma$ spectrum for the ${ }^{135} \mathrm{Xe}$ test case.
This validation is also done for two coupled differential equations (2) and (8) and does not show any discrepancies by using 100 time steps.

More of that we compared the results obtained for $10 \mathrm{~g}$ of uranium (235 and 238 with various isotopic vectors) to the results of MCNPX/CINDER [10] solving the depletion equation in a stochastic route. The results were fully comparable in spite of the different nuclear data used (GEF version used for the photofission yields, ENDF/B-VI for MCNPX instead of JEFF-3.1.1 Decay data libraries). The advantage of this automated script is to solve Bateman equations in few minutes and without convergence issues instead of the full Monte Carlo process.

\section{DELAYED $\gamma$ SPECTRA ANALYSIS}

We performed the calculation of delayed $\gamma(+\mathrm{X})$-spectra for all actinides of interest: ${ }^{235,238} \mathrm{U},{ }^{238,239,240,241,242} \mathrm{Pu}$ and ${ }^{241,242 \mathrm{~m}} \mathrm{Am}$ photon induced fissions for a $15 \mathrm{MeV}$ LINAC and a W conversion target. Fissile materials were supposed to be located at the center of the waste package. The final spectra is binned $(\Delta E=0.5$ or $1 \mathrm{keV})$ and analyzed above an energy threshold of $E_{\gamma}>1.3 \mathrm{MeV}$ (because of their rather high attenuation through the concrete in the waste package). A second filter (fixed by the user) concerns the minimum intensity of $\gamma$-lines $\frac{s_{\gamma i}}{\max \left(s_{\gamma i}\right)}>1 \%$, see figure 5 for the ${ }^{235} \mathrm{U}(\gamma, \mathrm{f})$ delayed $\gamma$-spectrum:

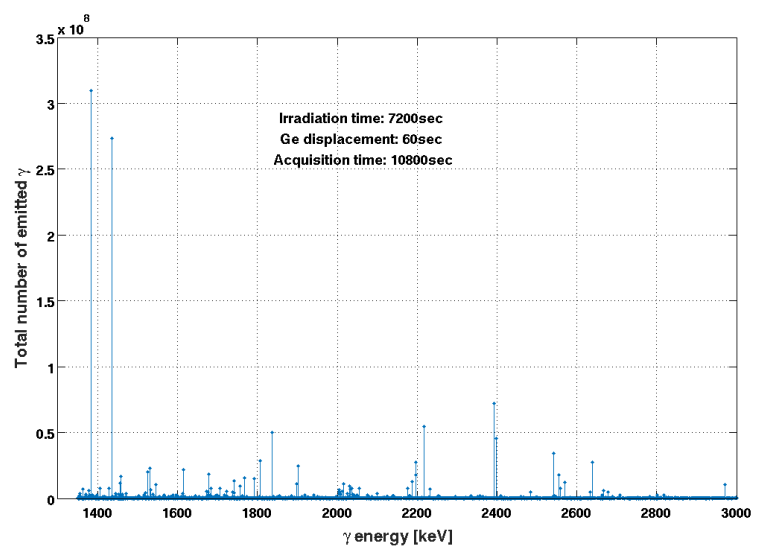

Fig. 5. Delayed $\gamma$ spectrum for ${ }^{235} \mathrm{U}$ photofissions integrated over the acquisition time.

The goal of the automated script is to distinguish the amount of each isotope in the fissile material. Taking profit that independent fission yields vary from a fissile system to another one, we developed a fully automated procedure to help the user to select specific $\gamma$ emissions. In order to avoid efficiency corrections in the final HPGe spectrum analyses, we decided to look for $\gamma$-line couples labeled 1 and 2 in a rather small energy window. The user has to specify two energy bands limits, for example $5 \mathrm{keV}<E_{\gamma 2}-E_{\gamma 1}<60 \mathrm{keV}$. The script will then look for all the $\gamma$ line couples that can distinguish uranium from plutonium sliding this energy window on the whole spectra. The final fitting parameter is the 
discriminant factor which is fixed to the emission line ratio $\frac{s_{\gamma 1}}{s_{\gamma 2}}>50 \%$ or $\frac{s_{\gamma 2}}{s_{\gamma 1}}>50 \%$.

We studied the particular case involving a fissile material made of $1 \mathrm{~g}$ of depleted uranium mixed with $10 \mathrm{~g}$ of plutonium (isotopic vector calculated at $40 \mathrm{GWd}-\mathrm{PWR} / \mathrm{t}_{\mathrm{HM}}$ ). The automated script DEGAS found more than $100 \gamma$-lines couples allowing distinguishing uranium from plutonium in the waste package. The figure 6 shows a zoom in the $1590-1670 \mathrm{keV}$ region. The bar histogram represents the plutonium and uranium emissions and the dashed lines symbolize the calculated and selected ratios of intensities for uranium and plutonium:

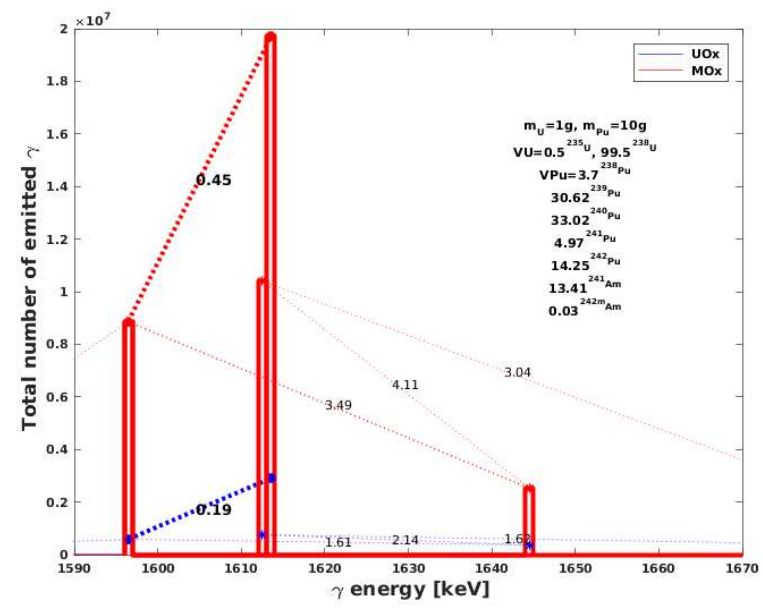

Fig. 6. Superimposed uranium (1g) and plutonium (10g) delayed $\gamma$-spectra after $2 \mathrm{~h}$ for irradiation ( $15 \mathrm{MeV}-\mathrm{W}$ conversion target) and $3 \mathrm{~h}$ for the acquisition time.

In figure 6 , the first $\gamma$ lines allowing to distinguish the uranium to the plutonium materials in this configuration are the following:

- $\quad E_{\gamma 1}=1596.7 \mathrm{keV}$ corresponding to the decay emission of the ${ }^{104} \mathrm{Tc}$,

- $\quad E_{\gamma 2}=1613.8 \mathrm{keV}$ corresponding to the decay emission of the ${ }^{134} \mathrm{I}$.

As shown in the figure 6 , the respective emission ratio prediction is:

- $\quad \frac{s_{\gamma 1}}{s_{\gamma_{2}}}=0.194$ for uranium,

- $\quad \frac{s_{\gamma 1}}{s_{\gamma 2}}=0.449$ for plutonium.

The automated selection of $\gamma$-ray will help the user to proceed to the second step of the analysis which consists in performing the transport of this few number of $\gamma$ through MCNPX from the fissile area to the HPGe detectors. Finally the goal will be to optimize the whole facility (irradiation versus acquisition time, on-line spectrum analysis for the characterization of the fissile isotopes involved in the nuclear waste package).

\section{CONCLUSION}

We proposed a simplified but robust and verified tool DEGAS to help the fine conception and the development of a nuclear material characterization facility by using photon interrogation techniques. It is based on the delayed $\gamma$-spectra analysis following fission events.

More of that, we proposed an automated procedure to estimate the amount and to give insights on the isotopic vector of fissile materials in a waste package. Results showed more than 100 discriminant $\gamma$-ray couples within few minutes of computer calculation time. This script can also be applied to delayed neutron detection in coincidence with the $\gamma$ lines.

All those calculation results suffer from nuclear data uncertainties (photofission cross sections, independent fission yields, decay data) that can be propagated in a further work, if needed.

To be complete, a necessary validation step versus an experimental actual facility will be set up in the following years at CEA.

\section{REFERENCES}

[1] Bateman, Harry. "The solution of a system of differential equations occurring in the theory of radioactive transformations." In Proc. Cambridge Philos. Soc, vol. 15, no. pt V, pp. 423- 427, 1910.

[2] M.A. Kellett, O. Bersillon, and R.W Mills, "The JEFF-3.1/-3.1.1 radioactive decay data and fission yields sub-libraries, JEFF Report 20", ISBN 978-92-64-99087-6, COECD/NEA 2009.

[3] MCNPX 2.7.0, "Monte Carlo N-Particle Transport Code System for Multiparticle and High Energy Applications", RSICC code package C740, (Radiation Safety Information Computational Center, ORNL, Oak Ridge,TN).

[4] M.B. Chadwick, et al., Nuclear Data Sheets 112 (2011) 2887-2996.

[5] https://tendl.web.psi.ch/tendl_2015/tendl2015.html

[6] K. H. Schmidt, B. Jurado, C. Amouroux, and C. Schmitt, Nuclear Data Sheets 131 (2016) 107.

[7] U. Brosa, S. Grossmann, and A. Muller, Phys. Rep. 197, 167 (1990).

[8] A. Tsilanizara, et al., "DARWIN: an Evolution Code System for a Large Range of Applications”, J. Nucl. Sci. Technol., Supplement, 1, pp. 845$849,2000$.

[9] MATLAB ${ }^{\circ}$ 9.2.0.538062 (R2017a). Natick, Massachusetts: The MathWorks Inc., 2010.

[10] Joe W. Durkee, et al., "Delayed-gamma signature calculation for neutron-induced fission and activation using MCNPX, Part I: Theory", Progress in Nuclear Energy 51 (2009) 813-827. 